BULLETIN OF THE

AMERICAN MATHEMATICAL SOCIETY

Volume 79, Number 3, May 1973

\title{
PERIODIC AND HOMOGENEOUS STATES ON A VON NEUMANN ALGEBRA. III
}

\author{
BY MASAMICHI TAKESAKI ${ }^{1}$ \\ Communicated by Jacob E. Feldman, September 12, 1972
}

In this paper, we will show with a fairly complete proof that most of the results in [10] hold for homogeneous periodic states on a factor without the assumption of inner homogeneity. As an application, we will see that nonisomorphic ergodic automorphisms $\tilde{\theta}$ of $\mathscr{Z}_{0}$ give rise to nonisomorphic factors $\mathscr{R}\left(\mathscr{M}_{0}, \theta\right)$ of type III. We keep most of the terminology and the notations in [9] and [10].

We consider an arbitrary pair of homogeneous periodic states $\varphi$ and $\psi$ on a factor $\mathscr{M}$ of the same period, say $T>0$. Let $\kappa=e^{-2 \pi / T}, 0<\kappa<1$. We denote by $\mathscr{M}_{n}^{\varphi, \psi}$ the set of all $x \in \mathscr{M}$ such that $\rho_{t}(x)=\kappa^{i n t} x, t \in \boldsymbol{R}$, which was denoted by $\mathscr{V}_{n}$ in [10]. With this alternation of the notation, we first note that Lemmas 1 through 6 remain valid without the assumption of inner homogeneity. Since $\mathscr{M}_{0}^{\varphi}$ and $\mathscr{M}_{0}^{\psi}$ are no longer factors, we have to analyze more carefully the relation between $\mathscr{M}_{0}^{\varphi}, \mathscr{M}_{0}^{\varphi, \psi}$ and $\mathscr{M}_{0}^{\psi}$. We denote by $\mathscr{Z}_{0}^{\varphi}$ and $\mathscr{Z}_{0}^{\psi}$ the center of $\mathscr{M}_{0}^{\varphi}$ and $\mathscr{M}_{0}^{\psi}$ respectively, and by $u_{\varphi}$ and $u_{\psi}$ the isometries in $\mathscr{M}_{1}^{\varphi}$ and $\mathscr{M}_{1}^{\psi}$ respectively which give rise to isomorphisms $\theta_{\varphi}$ and $\theta_{\psi}$ of $\mathscr{M}_{0}^{\varphi}$ and $\mathscr{M}_{0}^{\psi}$ onto $e_{\varphi} \mathscr{M}_{0}^{\varphi} e_{\varphi}$ and $e_{\psi} \mathscr{M}_{0}^{\psi} e_{\psi}$ respectively, where $e_{\varphi}=u_{\varphi} u_{\varphi}^{*}$ and $e_{\psi}=u_{\psi} u_{\psi}^{*}$. We also denote by $\tilde{\theta}_{\varphi}$ and $\tilde{\theta}_{\psi}$ the automorphisms of $\mathscr{Z}_{0}^{\varphi}$ and $\mathscr{Z}_{0}^{\psi}$ induced by $\theta_{\varphi}$ and $\theta_{\psi}$ respectively. Since $\mathscr{M}$ is a factor, we know from [9, Proposition 9] that $\tilde{\theta}_{\varphi}$ and $\tilde{\theta}_{\psi}$ are both ergodic.

LEMMA 1. For each $n \in Z$, we have

$$
\mathscr{M}_{n-1}^{\varphi, \psi}=u_{\varphi}^{*} \mathscr{M}_{n}^{\varphi, \psi} \text { and } \mathscr{M}_{n+1}^{\varphi, \psi}=\mathscr{M}_{n}^{\varphi, \psi} u_{\psi} .
$$

Proof. From [10, Lemma 5], it follows that $\mathscr{M}_{n-1}^{\varphi, \psi} \supset u_{\varphi}^{*} \mathscr{M}_{n}^{\varphi, \psi}$; so

$$
\mathscr{M}_{n-1}^{\varphi, \psi}=u_{\varphi}^{*} u_{\varphi} \mathscr{M}_{n-1}^{\varphi, \psi} \subset u_{\varphi}^{*} \mathscr{M}_{n}^{\varphi, \psi} \subset \mathscr{M}_{n-1}^{\varphi, \psi} \text {. }
$$

Hence we get $\mathscr{M}_{n-1}^{\varphi, \psi}=u_{\varphi}^{*} \mathscr{M}_{n}^{\varphi, \psi}$. By symmetry, the assertion for $u_{\psi}$ follows. Q.E.D.

LeMma 2. For any nonzero projections $p \in \mathscr{M}_{0}^{\varphi}$ and $q \in \mathscr{M}_{0}^{\psi}$, we have

$$
p \mathscr{M}_{n}^{\varphi, \psi} \neq\{0\} \text { and } \mathscr{M}_{n}^{\varphi, \psi} q \neq\{0\} .
$$

AMS (MOS) subject classifications (1970). Primary 46L10.

Key words and phrases. von Neumann algebra, modular automorphism group, periodic state, homogeneous state.

${ }^{1}$ This work is supported in part by NSF Grant No. GP-33696X. 
Proof. Let $\mathscr{I}_{n}$ be the set of all $x \in \mathscr{M}_{0}^{\varphi}$ with $x \mathscr{M}_{n}^{\varphi, \psi}=\{0\}$. By $[10$, Lemma 5], $\mathscr{I}_{n}$ is a $\sigma$-weakly closed ideal of $\mathscr{M}_{0}^{\varphi}$, so that there exists a projection $z_{n} \in \mathscr{Z}_{0}^{\varphi}$ such that $\mathscr{I}_{n}=\mathscr{M}_{0}^{\varphi} z_{n}$. If $x \in \mathscr{I}_{n}$, then $x \mathscr{M}_{n+1}^{\varphi, \psi}=x \mathscr{M}_{n}^{\varphi, \psi} u_{\psi}=\{0\}$ by (1), which means that $\mathscr{I}_{n} \subset \mathscr{I}_{n+1}$; so $z_{n} \leqq z_{n+1}$. We have

$$
\tilde{\theta}_{\varphi}\left(z_{n}\right) e_{\varphi} \mathscr{M}_{n+1}^{\varphi, \psi}=u_{\varphi} z_{n} u_{\varphi}^{*} \mathscr{M}_{n+1}^{\varphi, \psi}=u_{\varphi} z_{n} \mathscr{M}_{n}^{\varphi, \psi}=\{0\},
$$

so that $\tilde{\theta}_{\varphi}\left(z_{n}\right) e_{\varphi} \leqq z_{n+1}$; hence $\tilde{\theta}_{\varphi}\left(z_{n}\right) \leqq z_{n+1}$. Conversely, we have

$$
\tilde{\theta}_{\varphi}^{-1}\left(z_{n+1}\right) \mathscr{M}_{n}^{\varphi, \psi}=u_{\varphi}^{*} z_{n+1} u_{\varphi} \mathscr{M}_{n}^{\varphi, \psi} \subset u_{\varphi}^{*} z_{n+1} \mathscr{M}_{n+1}^{\varphi, \psi} \subset\{0\} .
$$

Hence we get $\tilde{\theta}_{\varphi}^{-1}\left(z_{n+1}\right) \leqq z_{n}$, so that $z_{n+1} \leqq \tilde{\theta}_{\varphi}\left(z_{n}\right)$. Thus we have $z_{n+1}=\tilde{\theta}_{\varphi}\left(z_{n}\right)$. Hence $z_{n} \leqq \tilde{\theta}_{\varphi}\left(z_{n}\right)$. The equality $\varphi\left(z_{n}\right)=\varphi \circ \tilde{\theta}_{\varphi}\left(z_{n}\right)$ implies that $z_{n}$ must be either 0 or 1 . Since $\mathscr{M}_{n}^{\varphi, \psi} \neq\{0\}$, we have $z_{n}=0$. Hence $p \mathscr{M}_{n}^{\varphi, \psi} \neq\{0\}$. By symmetry, $\mathscr{M}_{n}^{\varphi, \psi} q \neq\{0\}$. Q.E.D.

LEMMA 3. Let $v_{1}$ and $v_{2}$ be partial isometries in $\mathscr{M}_{n}^{\varphi, \psi}$ with initial projections $q_{1}, q_{2}$ and final projections $p_{1}, p_{2}$ respectively. Then the following statements are equivalent:

(i) $p_{1}$ and $p_{2}$ are centrally orthogonal in $\mathscr{M}_{0}^{\varphi}$, i.e. $p_{1} \mathscr{M}_{0}^{\varphi} p_{2}=\{0\}$;

(ii) $q_{1}$ and $q_{2}$ are centrally orthogonal in $\mathscr{M}_{0}^{\psi}$, i.e. $q_{1} \mathscr{M}_{0}^{\psi} q_{2}=\{0\}$.

Proof. By symmetry, we have only to prove (i) $\Rightarrow$ (ii). Suppose $q_{1} \mathscr{M}_{0}^{\psi} q_{2}$ $\neq\{0\}$. Let $x$ be an element in $\mathscr{M}_{0}^{\psi}$ with $q_{1} x q_{2} \neq\{0\}$. We have then $v_{1}^{*} v_{1} x v_{2}^{*} v_{2} \neq 0$, so that $v_{1} x v_{2}^{*} \neq 0$. Hence $p_{1} v_{1} x v_{2}^{*} p_{2}=v_{1} x v_{2}^{*} \neq 0$. But this is impossible because $v_{1} x v_{2}^{*}$ is in $\mathscr{M}_{0}^{\varphi}$ by $[\mathbf{1 0}$, Lemma 5]. Q.E.D.

Suppose $\left\{v_{i}\right\}_{i \in I}$ is a maximal family of partial isometries in $\mathscr{M}_{n}^{\varphi, \psi}$ such that the initial projections $q_{i}=v_{i}^{*} v_{i}$ are centrally orthogonal in $\mathscr{M}_{0}^{\psi}$. Let $p_{i}=v_{i} v_{i}^{*}$. By Lemma $3,\left\{p_{i}\right\}_{i \in I}$ are centrally orthogonal in $\mathscr{M}_{0}^{\varphi}$. Hence $v=\sum_{i \in I} v_{i}$ is a partial isometry in $\mathscr{M}_{n}^{\varphi, \psi}$. Let $p=v v^{*}$ and $q=v^{*} v$. By Lemma 3, we conclude that the central supports of $p$ and $q$ in $\mathscr{M}_{0}^{\varphi}$ and $\mathscr{M}_{0}^{\psi}$ are both the identity. Therefore, there exists an isomorphism $\sigma_{v}$ of $\mathscr{Z}_{0}^{\psi}$ onto $\mathscr{Z}_{0}^{\varphi}$ such that

$$
\sigma_{v}(a) p=v a v^{*}, \quad a \in \mathscr{Z}_{0}^{\psi} .
$$

LEMMA 4. For every projection $f \in \mathscr{Z}_{0}^{\psi}, \sigma_{v}(f)$ is characterized as the smallest projection e $\in \mathscr{Z}_{0}^{\varphi}$ such that $\mathscr{M}_{n}^{\varphi, \psi} f=\mathscr{M}_{n}^{\varphi, \psi} f$.

PROOF. Let $e$ be the smallest projection in $\mathscr{Z}_{0}^{\varphi}$ with $e \mathscr{M}_{n}^{\varphi} f=\mathscr{M}_{n}^{\varphi, \psi} f$. We have then $e v f=v f$, so that

$$
\sigma_{v}(f) p=v f v^{*}=e v f v^{*} e=e \sigma_{v}(f) p e=\sigma_{v}(f) e p .
$$

Hence $\left(\sigma_{v}(f)-\sigma_{v}(f) e\right) p=0$. Since the central support of $p$ is 1 , we have $\sigma_{v}(f)=\sigma_{v}(f) e$; that is, $\sigma_{v}(f) \leqq e$. If $e-\sigma_{v}(f) \neq 0$, then there exists an $x \in \mathscr{M}_{n}^{\varphi, \psi}$ with $\left[e-\sigma_{v}(f)\right] x f=x \neq 0$. Let $x=w h=k w$ be the left and right polar decomposition of $x$. As in the arguments (8) in [19], $w \in \mathscr{M}_{n}^{\varphi, \psi}$. 
By the choice of $x$, we have $w w^{*} \leqq e-\sigma_{v}(f)$ and $w^{*} w \leqq f$. On the other hand, we have $v f=\sigma_{v}(f) v f$, so that $(v f)(v f)^{*} \leqq \sigma_{v}(f)$ and $(v f)^{*}(v f)=f v^{*} v f$ $=f q$. Hence the central support of $(v f)^{*}(v f)$ in $\mathscr{M}_{0}^{\psi}$ is $f$. But this is impossible by Lemma 3 because the central supports of $(v f)(v f)^{*}$ and $w w^{*}$ in $\mathscr{M}_{0}^{\varphi}$ are orthogonal. Thus we get $\sigma_{v}(f)=e$. Q.E.D.

Therefore, the isomorphism $\sigma_{v}$ does not depend on the choice of $v$, but only on $n \in Z$; so we denote it by $\sigma_{n}$.

LEMMA 5. For each $n \in Z$, we have

$$
\sigma_{n} \circ \tilde{\theta}_{\psi}=\sigma_{n+1}=\tilde{\theta}_{\varphi} \circ \sigma_{n}
$$

Proof. Let $f$ be an arbitrarily fixed projection in $\mathscr{Z}_{0}^{\psi}$. Let $e_{n}=\sigma_{n}(f) \in \mathscr{Z}_{0}^{\varphi}$. We have then

$$
\begin{aligned}
e_{n+1} u_{\varphi} \mathscr{M}_{n}^{\varphi, \psi} f & =u_{\varphi} \mathscr{M}_{n}^{\varphi, \psi} f, \\
u_{\varphi}^{*} e_{n+1} u_{\varphi} \mathscr{M}_{n}^{\varphi, \psi} f & =\mathscr{M}_{n}^{\varphi, \psi} f .
\end{aligned}
$$

Hence we have $\tilde{\theta}_{\varphi}^{-1}\left(e_{n+1}\right) \geqq e_{n}$; equivalently, $e_{n+1} \geqq \tilde{\theta}_{\varphi}\left(e_{n}\right)$.

On the other hand, putting $z=1-\tilde{\theta}_{\varphi}\left(e_{n}\right)$, we have

$$
\begin{aligned}
u_{\varphi}^{*} z u_{\varphi} \mathscr{M}_{n}^{\varphi, \psi} f & =\left(1-e_{n}\right) \mathscr{M}_{n}^{\varphi, \psi} f=\{0\} \\
z e_{\varphi} \mathscr{M}_{n+1}^{\varphi, \psi} f & =z u_{\varphi} \mathscr{M}_{n}^{\varphi, \psi} f=u_{\varphi} u_{\varphi}^{*} z u_{\varphi} \mathscr{M}_{n}^{\varphi, \psi} f=\{0\} .
\end{aligned}
$$

Hence we have $z e_{\varphi} \leqq\left(1-e_{n+1}\right)$; so $z \leqq 1-e_{n+1}$. Therefore we get $\tilde{\theta}_{\varphi}\left(e_{n}\right) \geqq e_{n+1}$. Thus we have $e_{n+1}=\tilde{\theta}_{\varphi}\left(e_{n}\right)$; that is, $\tilde{\theta}_{\varphi} \circ \sigma_{n}(f)=\sigma_{n+1}(f)$ for every projection $f \in \mathscr{Z}_{0}^{\psi}$, which means that $\sigma_{n+1}=\tilde{\theta}_{\varphi} \circ \sigma_{n}$.

By symmetry, the other half of our assertion follows. Q.E.D.

COROLlaRY 6. The ergodic automorphisms $\tilde{\theta}_{\varphi}$ of $\mathscr{Z}_{0}^{\varphi}$ and $\tilde{\theta}_{\psi}$ of $\mathscr{Z}_{0}^{\psi}$ are isomorphic.

LEMMA 7. If $v$ is a partial isometry in $\mathscr{M}_{n}^{\varphi, \psi}$ such that the initial projection $q=v^{*} v$ and the final projection $p=v v^{*}$ have the central support 1 , then we have

$$
p^{\natural}=\alpha \kappa^{n} \sigma_{n}\left(q^{\natural}\right),
$$

where $\alpha$ is the real number defined in [10].

Proof. Consider a faithful state $\varphi \circ \sigma_{n}$ on $\mathscr{Z}_{0}^{\psi}$. Then $\varphi \circ \sigma_{n} \circ \tilde{\theta}_{\psi}=\varphi \circ \tilde{\theta}_{\varphi} \circ \sigma_{n}$ $=\varphi \circ \sigma_{n}$, so that $\varphi \circ \sigma_{n}$ is $\tilde{\theta}_{\psi}$-invariant; hence $\varphi \circ \sigma_{n}$ is a scalar multiple of $\psi$ on $\mathscr{Z}_{0}^{\psi}$ by the ergodicity of $\tilde{\theta}_{\psi}$. But $\varphi \circ \sigma_{n}$ and $\psi$ are both states, so that $\varphi \circ \sigma_{n}=\psi$ on $\mathscr{Z}_{0}^{\psi}$.

We have next, for every $a \in \mathscr{Z}_{0}^{\psi}$, 


$$
\begin{aligned}
\psi\left(a \sigma_{n}^{-1}\left(p^{\natural}\right)\right) & =\varphi\left(\sigma_{n}\left(a \sigma_{n}^{-1}\left(p^{\natural}\right)\right)\right)=\varphi\left(\sigma_{n}(a) p^{\natural}\right) \\
& =\varphi\left(\sigma_{n}(a) p\right)=\varphi\left(v a v^{*}\right) \\
& =\alpha \kappa^{n} \psi\left(a v^{*} v\right) \quad \text { by [10, Lemma 4] } \\
& =\alpha \kappa^{n} \psi(a q)=\alpha \kappa^{n} \psi\left(a q^{\natural}\right) .
\end{aligned}
$$

Thus, we get $\sigma_{n}^{-1}\left(p^{\natural}\right)=\alpha \kappa^{n} q^{\natural}=\alpha \kappa^{n} q^{\natural}$, equivalently $p^{\natural}=\alpha \kappa^{n} \sigma_{n}\left(q^{\natural}\right)$.

Making use of the similar arguments as in Lemma 2, we conclude the following:

LEMMA 8. If $p \in \mathscr{M}_{0}^{\varphi}$ and $q \in \mathscr{M}_{0}^{\varphi}$ are projections with central support $e$ and $f$ in $\mathscr{M}_{0}^{\varphi}$ and $\mathscr{M}_{0}^{\psi}$ respectively, then $p \mathscr{M}_{n}^{\varphi, \psi} q=\{0\}$ if and only if $e \sigma_{n}(f)$ $=0$.

Now, let $\left\{v_{i}\right\}_{i \in I}$ be a maximal family of partial isometries in $\mathscr{M}_{n}^{\varphi, \psi}$ such that the initial projections $q_{i}=v_{i}^{*} v_{i}$ and the final projections $p_{i}=v_{i} v_{i}^{*}$ are orthogonal respectively. Let $v=\sum_{i \in I} v_{i}, p=\sum_{i \in I} p_{i}$ and $q=\sum_{i \in I} q_{i}$. By Lemma 3 , the central supports of $p$ in $\mathscr{M}_{0}^{\varphi}$ and $q$ in $\mathscr{M}_{0}^{\psi}$ are respectively the identity. By maximality, we have $(1-p) \mathscr{M}_{n}^{\varphi, \psi}(1-q)=\{0\}$. Let $e$ and $f$ be the central supports of $p$ in $\mathscr{M}_{0}^{\varphi}$ and $q$ in $\mathscr{M}_{0}^{\psi}$ respectively. By Lemma 8, $e \sigma_{n}(f)=0$. On the other hand, we have $p^{\natural}=\alpha \kappa^{n} \sigma_{n}\left(q^{\natural}\right)$ by Lemma 7 . Hence $p^{\natural} \leqq \alpha \kappa^{n} \leqq 1$ if $n \geqq 1$. Hence we have $e=1$, so that $f=0$; so $q=1$. Hence $v$ must be an isometry if $n \geqq 1$. Similarly, if $n \leqq 0$, then $v$ is a co-isometry. For $n=0, v$ is unitary if and only if $\alpha=1$. Thus we reach the following conclusion:

THEOREM 9. If $\varphi$ and $\psi$ are homogeneous periodic states on a factor $\mathscr{M}$ with same period, then there exists isometries $u$ and $v$ in $\mathscr{M}$ such that

$$
\begin{aligned}
\psi(x) & =\varphi\left(u x u^{*}\right) / \varphi\left(u u^{*}\right), \\
\varphi(x) & =\psi\left(v x v^{*}\right) / \psi\left(v v^{*}\right), \quad x \in \mathscr{M}^{*} ; \\
p=u u^{*} & \in \mathscr{M}_{0}^{\varphi} \quad \text { and } q=v v^{*} \in \mathscr{M}_{0}^{\psi} .
\end{aligned}
$$

From this theorem, we can conclude that Theorems 8 through 10 in [10] hold for homogeneous periodic states $\varphi, \psi$ with the same period and/or for projections $p$ and $q$ with uniform relative dimensions.

Let $\mathscr{F}$ be a hyperfinite $\mathrm{II}_{1}$-factor and $\mathscr{A}=L^{\infty}(0,1)$. Let $\mathscr{M}_{0}=\mathscr{F} \otimes \mathscr{A}$. For $0<\kappa<1$, we choose a projection $f \in \mathscr{F}$ with $\tau(f)=\kappa$, where $\tau$ is the canonical trace of $\mathscr{F}$. Let $\theta$ be a fixed isomorphism of $\mathscr{F}$ onto $f \mathscr{F} f$. For each $\sigma \in \operatorname{Aut}(\mathscr{F})$, let $\theta_{\sigma}=\theta \circ \sigma$. Let $\tilde{\theta}$ be an ergodic automorphism of $\mathscr{A}$ with invariant faithful normal state $\mu$. Changing $\tilde{\theta}$ under an automorphism of $\mathscr{A}$, we may assume that $\mu$ is given by the Lebesgue measure on $(0,1)$. Let $\varphi_{0}=\tau \otimes \mu$. We obtain then a factor $\mathscr{R}\left(\mathscr{M}_{0}, \theta_{\sigma} \otimes \tilde{\theta}, \varphi_{0}\right)$ as described in [9]. We denote it by $\mathscr{M}(\kappa, \sigma, \tilde{\theta})$. 
THEOREM 10. We choose $\sigma_{1}, \sigma_{2} \in \operatorname{Aut}(\mathscr{F})$ and ergodic automorphisms $\tilde{\theta}_{1}$ and $\tilde{\theta}_{2}$ of $\mathscr{A}$ and fix $\kappa$. A necessary and sufficient condition for $\mathscr{M}\left(\kappa, \sigma_{1}, \tilde{\theta}_{1}\right)$ $\cong \mathscr{M}\left(\kappa, \sigma_{2}, \tilde{\theta}_{2}\right)$ is that

(i) $\tilde{\theta}_{1}$ and $\tilde{\theta}_{2}$ are isomorphic as ergodic automorphisms of $\mathscr{A}$;

(ii) there exist a projection $p$ in $\mathscr{F}$ with $p \geqq f$, a partial isometry $w$ in $\mathscr{F}$ and an isomorphism $\rho$ of $\mathscr{F}$ onto p $\mathscr{F} p$ such that

$$
\begin{aligned}
w \theta \circ \sigma_{1} \circ \rho(x) w^{*} & =\rho \circ \theta \circ \sigma_{2}(x) ; \\
\theta \circ \sigma_{1} \circ \rho(x) & =w^{*} \rho \circ \theta \circ \sigma_{2}(x) w, \quad x \in \mathscr{M} .
\end{aligned}
$$

Furthermore, if $\tilde{\theta}_{1}$ and $\tilde{\theta}_{2}$ have no point spectrum other than 1 , then the isomorphy $\mathscr{M}\left(\kappa_{1}, \sigma_{1}, \tilde{\theta}_{1}\right) \cong \mathscr{M}\left(\kappa_{2}, \sigma_{2}, \tilde{\theta}_{2}\right)$ implies that $\kappa_{1}=\kappa_{2}$ as well as (i) and (ii).

AcKNowledgement. The author would like to thank Professor H. Araki for enjoyable discussions on the subject of this paper; Dr. A. Connes for his kindness informing the author of his new results on which this work depends; Professor H. A. Dye for his constant encouragement; and the members of the functional analysis seminar at Queen's University where the related topics were extensively discussed.

\section{REFERENCES}

1. A. Connes, Un nouvel invariant pour les algèbre de von Neumann, C. R. Acad. Sci. Paris Sér. A-B 273 (1971), A900-A903.

2. - Calcul des deux invariants d'Araki et Woods par la théorie de Tomita et Takesaki, C. R. Acad. Sci. Paris Sér. A-B 274 (1972), A175-A177.

3. - Groupe modulairé d'une algèbre de von Neumann de genre denombrable, C.R. Acad. Sci. Paris Sér. A-B 274 (1972), A1923-A1926.

4. - États presque périodiques sur une algèbre de von Neumann, C. R. Acad. Sci. Paris Sér. A-B 274 (1972), A1402-A1405.

5. - A letter to the author, dated June 17, 1972.

6. J. Dixmier, Les algèbres d'opérateurs dans l'espace hilbertien (Algèbre de von Neumann), Cahiers scientifiques, fasc. 25, Gauthier-Villars, Paris, 1957. MR 20 \# 1234.

7. E. Størmer, Spectra of states, and asymptotically abelian $C^{*}$-algebras (to appear).

8. M. Takesaki, Tomita's theory of modular Hilbert algebras and its applications, Lecture Notes in Math., vol. 128, Springer-Verlag, Berlin and New York, 1970. MR 42 \# 5061.

9. - Periodic and homogeneous states on a von Neumann algebra. I. Bull. Amer. Math. Soc. 79 (1973), 202-206.

10. Math. Soc. 79 (1973), 416-420.

11. - The structure of a von Neumann algebra with a homogeneous periodic state, Acta Math. (to appear).

Department of Mathematics, University of California, Los Angeles, California 90024 